Pacific Journal of Mathematics

INVARIANT SUBSPACES AND OPERATORS OF CLASS (S 


\section{INVARIANT SUBSPACES AND OPERATORS OF CLASS $(S)$}

\section{NORBERTo SALINAS}

Let $\mathscr{H}$ be an infinite dimensional separable complex Hilbert space, and let $\mathscr{L}(\mathscr{H})$ denote the algebra of all (bounded linear) operators on $\mathscr{H}$. This paper is concerned with a specific class of two-by-two operator matrices acting in the usual fashion on $\mathscr{H} \oplus \mathscr{H}$. An operator in $\mathscr{L}(\mathscr{H} \oplus \mathscr{H})$ will be said to be of class $(S)$ if it can be represented as a two by two operator matrix of the form

$$
\left[\begin{array}{cc}
A & V \\
-V^{*} & 0
\end{array}\right]
$$

where $V$ is a unilateral shift of infinite multiplicity on $\mathscr{H}$ and $A$ is an arbitrary operator in $\mathscr{L}(\mathscr{H})$.

In the present paper it is shown that the study of the operators of class $(S)$ arises naturally in connection with the invariant subspace problem. In particular, the question of whether an operator of class $(S)$ has a nontrivial invariant subspace is raised, and some significant results are obtained toward the solution of this problem.

Following [4] we shall denote by $(F)$ the set of all operators which cannot be written in the form $\lambda+K$, where $\lambda$ belongs to the complex field $\boldsymbol{C}$, and $K$ is in the ideal $\mathscr{K}$ of all compact operators. Brown and Pearcy in [4], Theorem 2 found, up to similarity, a standard form for operators in $(F)$. As a consequence of that theorem they showed ([4], Corollary 3.4) that every operator $T \in(F)$ is similar to an operator matrix of the form

$$
\left[\begin{array}{cc}
R & W \\
S & 0
\end{array}\right]
$$

acting on $\mathscr{H} \oplus \mathscr{H}$, where $W$ is an isometry of infinite deficiency (i.e. null $W^{*}$ is infinite dimensional).

Our first objective in this paper is to obtain a simplification in the representing matrix $(*)$ (up to similarity) of an operator in $(F)$. In this fashion, we prove (Theorem 1) that every operator $T \in(F)$ is similar to an operator matrix of the form

$$
\left[\begin{array}{ll}
A & V \\
B & 0
\end{array}\right],
$$

where $A, B \in \mathscr{L}(\mathscr{H})$ and $V$ is a unilateral shift of infinite multiplicity ([8]). 
We then show (Theorem 3) that, up to translation, every operator in $(F)$ is similar to an operator of the form$$
\left[\begin{array}{cc}
A & V \\
-C V^{*} & 0
\end{array}\right]
$$

acting on $\mathscr{X} \oplus \mathscr{C}$, where $V$ is a unilateral shift of infinite multiplicity, $A$ is in the class $(E D)$ of all operators whose spectrum is essentially disconnected ${ }^{1}$, and $A$ is invertible as well as the operators $C, V^{*} A V$ and $V^{*} A^{-1} V$. Since every compact operator has a proper invariant subspace ([1]), the last result produces an equivalent formulation of the general invariant subspace problem on Hilbert space, namely every operator on a Hilbert space has a nontrivial invariant subspace, if and only if the same property holds for every operator matrix of the form $\left({ }^{* *}\right)$, satisfying the conditions described above. It is by virtue of this result that operators of class $(S)$ make their appearance on the scene of the invariant subspace problem. In order to glimpse the relationship between the invariant subspace problem for operators in class $(S)$ and the general problem, let us observe the following trivial factorization of an operator of the form $(* *)$ :

$$
\left[\begin{array}{cc}
A & V \\
-C V^{*} & 0
\end{array}\right]=\left[\begin{array}{ll}
1 & 0 \\
0 & C
\end{array}\right]\left[\begin{array}{cc}
A & V \\
-V^{*} & 0
\end{array}\right]
$$

Thus, it would be of interest to obtain any information possible about operators in $(S)$, with a view to gaining more insight into the difficulties involved in the general invariant subspace problem for operators on Hilbert space.

In $\S 3$ we make some remarks concerning operators of class $(S)$, and in $\S 4$ we restrict our attention to a special kind of operators in class $(S)$, namely operators of the form

$$
\left[\begin{array}{cc}
P & V \\
-V^{*} & 0
\end{array}\right]
$$

where $P$ is any projection in $\mathscr{L}(\mathscr{C})$. These operators are not normal, in general (neither hyponormal; Lemma 3.1) and hence it is not a simple matter to find invariant subspaces for them. We use a deep algebraic result of Kaplansky ([9]) to prove that

$$
\left[\begin{array}{cc}
P & V \\
-V^{*} & 0
\end{array}\right]
$$

${ }^{1}$ An operator $T$ is said to belong to $(E D)([\mathbf{1 0}]$, Theorem 2$)$ if the polynomial hull $\hat{E}(T)$ of the essential spectrum $E(T)$ of $T$ is disconnected. 
has, in fact, nontrivial reducing subspaces, and even more with the techniques provided in [3] and [5] we show (Theorem 5) that

$$
\left[\begin{array}{cc}
P & V \\
-V^{*} & 0
\end{array}\right]
$$

is in the class $(E D)$.

Some properties of the class $(E D)$ were discussed in [10]. There, it is shown ([10], Theorem 7) that the class $(E D)$ is open in the uniform topology. It is also proved ([10], Theorem 8) that every operator $T \in(E D)$ has two complementary, infinite dimensional hyperinvariant subspaces ${ }^{2}$.

Thus, as a corollary of Theorem 5 (already mentioned) and the last remark, we conclude that, if $P$ is any projection in $\mathscr{L}(\mathscr{H})$, then there exists $\varepsilon>0$ such that for every $R \in \mathscr{L}(\mathscr{K})$, with $\|R\|<\varepsilon$ and every compact $K$, the operator

$$
\left[\begin{array}{cc}
P+R+K & V \\
-V^{*} & 0
\end{array}\right]
$$

has two complementary, infinite dimensional hyperinvariant subspaces.

2. Invariant subspaces via the standard form of Brown and Pearcy. Our main tool in the present section is a theorem of Brown and Pearcy that provides a particular representation for any operator in $(F)$, up to similarity, as a $3 \times 3$ operator matrix ([4], Theorem 2). It can be stated as follows:

Theorem (Brown and Pearcy). Let $T \in(F)$. Then there exists an invertible transformation $Z: \mathscr{Y} \rightarrow \mathscr{Y} \oplus \mathscr{K} \oplus \mathscr{K}$ such that

$$
Z T Z^{-1}=\left[\begin{array}{lll}
T_{11} & T_{12} & 0 \\
T_{21} & T_{22} & 1 \\
T_{31} & T_{32} & 0
\end{array}\right],
$$

where $T_{i j} \in \mathscr{C}(\mathscr{\mathscr { C }}), i=1,2,3 ; j=1,2$ and $\left\|T_{11}\right\| \leqq\|T\|$.

Lemma 2.1. Let $T_{i j} \in \mathscr{P}(\mathscr{H}), i=1,2,3 ; j=1,2$, and let

$$
\widetilde{T} \in \mathscr{L}(\mathscr{H} \oplus \mathscr{H} \oplus \mathscr{H})
$$

be defined by

${ }^{2}$ The basic properties of hyperinvariant subspaces are discussed in [6]. 


$$
\widetilde{T}=\left[\begin{array}{lll}
T_{11} & T_{12} & 0 \\
T_{21} & T_{22} & 1 \\
T_{31} & T_{32} & 0
\end{array}\right] .
$$

Then there exists a unitary transformation $J: \mathscr{H} \oplus \mathscr{H} \oplus \mathscr{H} \rightarrow \mathscr{H} \oplus \mathscr{H}$ such that

$$
J \widetilde{T} J^{-1}=\left[\begin{array}{ll}
A_{1} & V \\
A_{2} & 0
\end{array}\right],
$$

where $V$ is a unilateral shift of infinite multiplicity. Moreover, if $T_{31}=0$, then $A_{2}=T_{32} V^{*}$. On the other hand, if $T_{11}$ is invertible, then $\widetilde{T}$ is similar to an operator of the form

$$
\left[\begin{array}{cc}
A_{3} & V \\
A_{4} V^{*} & 0
\end{array}\right]
$$

Proof. Let $J_{0}: \mathscr{H} \oplus \mathscr{H} \rightarrow \mathscr{H}$ be any unitary transformation and let $\widehat{J}: \mathscr{H} \oplus \mathscr{H} \oplus \mathscr{H} \rightarrow \mathscr{H} \oplus \mathscr{H}$ be given by $\hat{J}=J_{0} \oplus 1$; hence $\hat{J}^{-1}=\hat{J}^{*}=J_{0}^{*} \oplus 1$. It follows easily that the $2 \times 2$ matrix $\widehat{J} \widetilde{T} \widehat{J}^{*}$ has the form

$$
\left[\begin{array}{cc}
B_{1} & W \\
B_{2} & 0
\end{array}\right]
$$

where

$$
B_{1}=J_{0}\left[\begin{array}{ll}
T_{11} & T_{12} \\
T_{21} & T_{22}
\end{array}\right] J_{0}^{*}, W=J_{0}\left[\begin{array}{l}
0 \\
1
\end{array}\right], B_{2}=\left[T_{31} T_{32}\right] J_{0}^{*} .
$$

Here, $\left[\begin{array}{l}0 \\ 1\end{array}\right]: \mathscr{H} \rightarrow \mathscr{H} \oplus \mathscr{H}$ and $\left[\begin{array}{ll}T_{31} & T_{32}\end{array}\right]: \mathscr{H} \oplus \mathscr{C} \rightarrow \mathscr{H}$ are the bounded linear transformations defined in the usual matricial fashion. Since

$$
W^{*}=\left[\begin{array}{ll}
0 & 1
\end{array}\right] J_{0}^{*},
$$

we see that

$$
W W^{*}=J_{0}\left[\begin{array}{l}
0 \\
1
\end{array}\right][0 \quad 1] J_{0}^{*}=J_{0}\left[\begin{array}{ll}
0 & 0 \\
0 & 1
\end{array}\right] J_{0}^{*} \cdot
$$

and

$$
W^{*} W=\left[\begin{array}{ll}
0 & 1
\end{array}\right] J_{0}^{*} J_{0}\left[\begin{array}{l}
0 \\
1
\end{array}\right]=1
$$

Therefore $W$ is an isometry, and $\operatorname{ran}\left(1-W W^{*}\right)\left(=\right.$ null $\left.W^{*}\right)$ is infinite dimensional. If $T_{31}=0$, 


$$
B_{2}=\left[\begin{array}{ll}
0 & T_{32}
\end{array}\right] J_{0}^{*}=T_{32}\left[\begin{array}{ll}
0 & 1
\end{array}\right] J_{0}^{*}=T_{32} W^{*} .
$$

Next we prove that there exists a unitary $U \in \mathscr{L}(\mathscr{H})$ such that $U W$ is a unilateral shift of infinite multiplicity. Let $\left\{e_{n}\right\}$ be an orthonormal basis of $\mathscr{H}$. Also let $\mathscr{C}=\operatorname{ran} W$. Then $\left\{f_{n}\right\}$, where $f_{n}=W e_{n}$, constitutes an orthonormal basis of $\mathscr{M}$. Let $\left\{g_{n}\right\}$ be an orthonormal basis of $\mathscr{l}^{\perp}$, and define $U \in \mathscr{L}(\mathscr{H})$ on the basis $\left\{f_{n}, g_{n}\right\}$ of $\mathscr{H}$ by $U f_{n}=e_{2 n}, U g_{n}=e_{2 n-1}, n=1,2, \cdots$ Let $V=U W$; by construction $V e_{n}=e_{2 n}$ and hence null $V^{*}$ is infinite dimensional and $\bigcap_{n=1}^{\infty} V^{n} \mathscr{C}=$ $\{0\}$. Then $V$ is a unilateral shift of infinite multiplicity. Now, the first part of the assertion follows by defining $J: \mathscr{H} \oplus \mathscr{H} \oplus \mathscr{H} \rightarrow$ $\mathscr{H} \oplus \mathscr{H}$ as

$$
J=\left[\begin{array}{ll}
U & 0 \\
0 & 1
\end{array}\right] \hat{J} \quad\left(=\left(U J_{0}\right) \oplus 1\right)
$$

Finally, if $T_{11}$ is invertible, the matrix

$$
\widetilde{S}=\left[\begin{array}{ccc}
1 & 0 & 0 \\
0 & 1 & 0 \\
-T_{31} T_{11}^{-1} & 0 & 1
\end{array}\right]
$$

is also invertible, and the operator $\widetilde{T}^{\prime}=\widetilde{S} \widetilde{T} \widetilde{S}^{-1}$ has the form

$$
\left[\begin{array}{lll}
* & * & 0 \\
* & * & 1 \\
0 & * & 0
\end{array}\right] \text {. }
$$

The proof is completed after applying the first part of the present lemma to the matrix $\widetilde{T}^{\prime}$.

THEOREM 1. Every operator $T$ in $(F)$ is similar to an operator matrix of the form

$$
\left[\begin{array}{ll}
A & V \\
B & 0
\end{array}\right]
$$

acting on $\mathscr{H} \oplus \mathscr{H}$, where $V$ is a unilateral shift of infinite multiplicity.

Proof. It follows directly from Lemma 2.1 and the theorem of Brown and Pearcy mentioned previously.

Lemma 2.2. Let $T \in(F)$ and let $M$ be the scalar operator entry matrix 


$$
M=\left[\begin{array}{rrr}
1 & 0 & 0 \\
0 & 2 & 1 \\
0 & -1 & 0
\end{array}\right]
$$

acting on $\mathscr{H} \oplus \mathscr{H} \oplus \mathscr{H}$. Then, for $|\lambda|>\|T\|$ there exist invertible transformations $Z_{\lambda}: \mathscr{H} \rightarrow \mathscr{H} \oplus \mathscr{H} \oplus \mathscr{H}$ and operator matrices $R_{\lambda} \in \mathscr{L}(\mathscr{H} \oplus \mathscr{H} \oplus \mathscr{H})$ of the form

$$
R_{\lambda}=\left[\begin{array}{lll}
* & * & 0 \\
* & * & 0 \\
0 & * & 0
\end{array}\right]
$$

satisfying the conditions:

(a) $Z_{\lambda}(T+\lambda) Z_{\lambda}^{-1}=R_{\lambda}+\lambda M$;

(b) $\left\|R_{\lambda}\right\| \leqq k<\infty$;

(c) the following limits exist (in the norm topology),

$$
\lim _{\lambda \rightarrow \infty} Z_{\lambda}=Z_{0}, \quad \lim _{\lambda \rightarrow \infty}(1 / \lambda) Z_{\lambda}^{-1}=Z_{1},
$$

and $Z_{1} Z_{0}=0$.

On the other hand, given $S \in \mathscr{L}(\mathscr{H})$ and a scalar operator entry matrix $N \in \mathscr{P}(\mathscr{H} \oplus \mathscr{H} \oplus \mathscr{H}), N \neq 1$, if there exist invertible transformations $Z_{\lambda}: \mathscr{H} \rightarrow \mathscr{H} \oplus \mathscr{H} \oplus \mathscr{H}$ and operators $R_{\lambda} \in \mathscr{L}(\mathscr{H} \oplus \mathscr{H} \oplus \mathscr{H})$ such that for every $|\lambda|>\|S\|$, conditions (a)(b) and (c) hold whenever $T$ and $M$ are replaced by $S$ and $N$ respectively, then $S \in(F)$ and $N$ is similar to $M$.

Proof. From the theorem of Brown and Pearcy, there exist an invertible transformation $Z: \mathscr{H} \rightarrow \mathscr{H} \oplus \mathscr{H} \oplus \mathscr{H}$ such that

$$
Z T Z^{-1}=\left[\begin{array}{lll}
T_{11} & T_{12} & 0 \\
T_{21} & T_{22} & 1 \\
T_{31} & T_{32} & 0
\end{array}\right],
$$

$T_{i, j} \in \mathscr{C}(\mathscr{C}), i=1,2,3, j=1,2,\left\|T_{11}\right\| \leqq\|T\|$. Thus, for $|\lambda|>\|T\|$, $T_{11}+\lambda$ is invertible and for such values of $\lambda$ the operator matrix

$$
X_{\lambda}=\left[\begin{array}{crr}
1 & 0 & 0 \\
0 & 1 & 0 \\
\left(T_{21}-\frac{T_{31}}{\lambda}\right)\left(T_{11}+\lambda\right)^{-1} & -1 & 1 / \lambda
\end{array}\right]
$$

is invertible in $\mathscr{Y}(\mathscr{Y} \oplus \mathscr{\mathscr { C }} \oplus)$; its inverse is

$$
X_{\lambda}^{-1}=\left[\begin{array}{ccc}
1 & 0 & 0 \\
0 & 1 & 0 \\
\left(T_{31}-\lambda T_{21}\right)\left(T_{11}+\lambda\right)^{-1} & \lambda & \lambda
\end{array}\right] .
$$


Now we define

$$
Z_{\lambda}=X_{\lambda} Z
$$

First, we note that

$$
\lim _{\lambda \rightarrow \infty} Z_{\lambda}=\left[\begin{array}{rrr}
1 & 0 & 0 \\
0 & 1 & 0 \\
0 & -1 & 0
\end{array}\right] Z=Z_{0}
$$

and

$$
\lim _{\lambda \rightarrow \infty}\left(\frac{1}{\lambda}\right) Z_{\lambda}^{-1}=Z^{-1}\left[\begin{array}{lll}
0 & 0 & 0 \\
0 & 0 & 0 \\
0 & 1 & 1
\end{array}\right]=Z_{1} .
$$

Therefore $Z_{1} Z_{0}=0$ and (c) is satisfied. In order to prove (a) we compute

$$
Z_{\lambda}(T+\lambda) Z_{\lambda}^{-1}=X_{\lambda} Z(T+\lambda) Z^{-1} X_{\lambda}^{-1}=R_{\lambda}+\lambda M
$$

where

$R_{\lambda}=\left[\begin{array}{ccc}T_{11} & T_{12} & 0 \\ T_{21}+\left(T_{31}-\lambda T_{21}\right)\left(T_{11}+\lambda\right)^{-1} & T_{22} & 0 \\ 0 & \frac{1}{\lambda} T_{32}+\left(T_{21}-\frac{T_{31}}{\lambda}\right)\left(T_{11}+\lambda\right)^{-1} T_{12}-T_{22} & 0\end{array}\right]$.

Now it is clear that (b) is also valid. Finally, to show the last assertion we divide the equation in part (a) by $\lambda$, and then we take limit when $\lambda$ tends to infinite to obtain

$$
Z_{0} S Z_{1}=N-1 \text {. }
$$

From (c) $(N-1)^{2}=0$. Since the same property holds for $M$, and $N \neq 1$, it follows that $M$ and $N$ are similar. The proof of the theorem is completed by observing that $N \in(F)$, and hence $S \in(F)$.

The following theorem is central to our purposes.

THEOREM 2. Let $T \in(F)$, then for $|\lambda|>\|T\|$ there exist invertible transformations $Y_{:}: \mathscr{C} \rightarrow \mathscr{C} \oplus \mathscr{H}$, and operators $B_{\lambda}, C_{\lambda} \in \mathscr{C}(\mathscr{H})$ such that

$$
Y_{\lambda}(T+\lambda) Y_{\lambda}^{-1}=\left[\begin{array}{cc}
B_{\lambda}+\lambda\left(1+V V^{*}\right) & \lambda V \\
-\left(C_{\lambda}+\lambda\right) V^{*} & 0
\end{array}\right],
$$

where $V$ is a unilateral shift of infinite multiplicity on $\mathscr{C}$; 


$$
\left\|B_{\lambda}\right\| \leqq m<\infty, \quad\left\|C_{\lambda}\right\| \leqq m<\infty
$$

Proof. We use the unitary transformation $J: \mathscr{H} \oplus \mathscr{H} \oplus \mathscr{H} \rightarrow$ $\mathscr{H} \oplus \mathscr{X}$ provided by Lemma 2.1. It is easy to check

$$
J\left[\begin{array}{rrr}
1 & 0 & 0 \\
0 & 2 & 1 \\
0 & -1 & 0
\end{array}\right] J^{*}=\left[\begin{array}{cc}
1+V V^{*} & V \\
-V^{*} & 0
\end{array}\right] .
$$

Now setting $Y_{\lambda}=J Z_{\lambda}$, we see that the present theorem follows from Lemma 2.2.

The above theorem has an interesting connection with the invariant subspace problem. The next result exhibits such a relationship more explicitly.

TheOREM 3. Every operator $T \in(F)$ can be written, up to similarity and translation by a complex number, as a $2 \times 2$ operator matrix acting on $\mathscr{H} \oplus \mathscr{H}$, of the form

$$
\left[\begin{array}{cc}
A & V \\
-C V^{*} & 0
\end{array}\right]
$$

where $V$ is a unilateral shift of infinite multiplicity, $A$ is an invertible operator of class (ED) and the operators $C, V^{*} A V$ and $V^{*} A^{-1} V$ are also invertible.

Proof. From Theorem 2, for $|\lambda|>\|T\|$, we can apply the similarity $Y_{2}$, and the translation by $\lambda$ to the operator $T$ to obtain

$$
T_{\lambda}=Y_{\lambda}(T+\lambda) Y_{\lambda}^{-1}=\left[\begin{array}{cc}
B_{\lambda}+\lambda\left(1+V V^{*}\right) & \lambda V \\
-\left(C_{\lambda}+\lambda\right) V^{*} & 0
\end{array}\right] .
$$

Let $S_{2}$, be the invertible operator

$$
S_{\lambda}=\left[\begin{array}{ll}
1 & 0 \\
0 & \lambda
\end{array}\right]
$$

Then

$$
S_{\lambda} T_{\lambda} S_{\lambda}^{-1}=\left[\begin{array}{cc}
B_{\lambda}+\lambda\left(1+V V^{*}\right) & V \\
-\lambda\left(C_{\lambda}+\lambda\right) V^{*} & 0
\end{array}\right] .
$$

From Theorem 2-(ii) it follows that for $|\lambda|>m, C_{\lambda}+\lambda$ is invertible. On the other hand we observe that $1+V V^{*}$ is a positive invertible operator whose essential spectrum is disconnected. Therefore, also from Theorem 2-(ii), it follows that, for $\lambda$ large enough, $B_{\lambda}+\lambda\left(1+V V^{*}\right)=$ $A_{\lambda}$ is an invertible operator of class $(E D)$. Let $\lambda_{0}$ be any complex 
number satisfying the above condition and $\left|\lambda_{0}\right|>\max (m,\|T\|)$ and set $A_{\lambda_{0}}=A, \lambda_{0}\left(C_{\lambda_{0}}+\lambda_{0}\right)=C$. Now it readily follows that $V^{*} A V$ and

$$
\left[\begin{array}{cc}
A & V \\
-C V^{*} & 0
\end{array}\right]
$$

are invertible, and hence $V^{*} A^{-1} V$ is also invertible.

REMARK. Some other comments are also pertinent with respect to the connection between Theorem 2 and the invariant subspace problem. If in the matrix

$$
T_{\lambda}=\left[\begin{array}{cc}
B_{\lambda}+\lambda\left(1+V V^{*}\right) & \lambda V \\
-\left(C_{\lambda}+\lambda\right) V^{*} & 0
\end{array}\right]
$$

of Theorem 2-(i) $B_{\lambda}$ and $C_{\lambda}$ are compact, for some $\lambda$, then $T_{\lambda}$ is polynomially compact and hence ([2]) it has a proper invariant subspace. On the other hand, if $B_{\lambda}$ vanishes for some $\lambda$, then $T_{\lambda}$ has an obvious invariant subspace, namely the range of the projection

$$
\left[\begin{array}{cc}
1-V V^{*} & 0 \\
0 & 0
\end{array}\right]
$$

However, if for some $\lambda_{1}, C_{\lambda_{1}}=0$ we don't know, a priori, anything about the invariant subspaces of $T_{\lambda_{1}}$. Nevertheless, some particular cases are worth noting. Set

$$
B=B_{\lambda_{1}} / \lambda_{1}
$$

and

$$
T_{1}=T_{\lambda_{1}} \lambda_{1}=\left[\begin{array}{cc}
1+V V^{*}+B & V \\
-V^{*} & 0
\end{array}\right] .
$$

It is easy to see that if $\overline{\operatorname{ran} B}$ is a proper subspace, then so is $\overline{\operatorname{ran}\left(T_{1}-1\right)}$, and hence $T_{1}$ has a nontrivial invariant subspace. on the other hand, if $\overline{\operatorname{ran} B}=\mathscr{H}$, but $B$ has a proper invariant subspace $\mathscr{C l}$ containing ran $B V V^{*}$, then the operator $T_{1}$ again has a proper invariant subspace. In fact, if $P$ is the projection onto $\mathscr{C l}$, the closure of

$$
\operatorname{ran}\left[\begin{array}{cc}
V V^{*} & P \\
-V^{*} & 0
\end{array}\right]
$$

is proper and it is an invariant subspace of $T_{1}$ because of the relation

$$
\left[\begin{array}{cc}
1+V V^{*} B & V \\
-V^{*} & 0
\end{array}\right]\left[\begin{array}{cc}
V V^{*} & P \\
-V^{*} & 0
\end{array}\right]=\left[\begin{array}{cc}
V V^{*} & P \\
-V^{*} & 0
\end{array}\right]\left[\begin{array}{cr}
V V^{*} & P \\
B V V^{*} & B P+1
\end{array}\right] .
$$


In any case, if $B$ is arbitrary, the invariant subspace problem for operators of the form

$$
\left[\begin{array}{cc}
1+V V^{*}+B & V \\
-V^{*} & 0
\end{array}\right]
$$

seems to be hard, even though it is apparently simpler than the general invariant subspace problem. This is one of the reasons that operators of the form

$$
\left[\begin{array}{cc}
A & V \\
-V^{*} & 0
\end{array}\right]
$$

where, as above, $V$ is a unilateral shift of infinite multiplicity, are interesting objects of investigation.

3. Some properties of operators of class $(S)$. In this section we make some remarks concerning operators of the form

$$
\left[\begin{array}{cc}
A & V \\
-V^{*} & 0
\end{array}\right]
$$

acting on $\mathscr{H} \oplus \mathscr{H}$. Even though some of the following results about these operators are also valid when $V$ is replaced by any isometry in $\mathscr{L}(\mathscr{H})$, we prefer to restrict our considerations to operator matrices of class $(S)$, that is, to the case in which $V$ is a fixed unilateral shift of infinite multiplicity on $\mathscr{Y}$.

In the next two results we summarize some simple but useful facts concerning operators of class $(S)$. We omit their proofs since they consist of straightforward verifications.

Lemma 3.1. The operator

$$
\left[\begin{array}{cc}
A & V \\
-V^{*} & 0
\end{array}\right]
$$

is normal (hyponormal) if and only if $A$ is normal (hyponormal) and $\left(A+A^{*}\right) V=0$.

Lemma 3.2. All the diagonal invariant (reducing) projections of

$$
\left[\begin{array}{cc}
A & V \\
-V^{*} & 0
\end{array}\right]
$$

are of the form

$$
\left[\begin{array}{cc}
P & 0 \\
0 & V^{*} P V
\end{array}\right]
$$


where $P$ is an invariant (reducing) projection of $A$ commuting with $V V^{*}$.

The following lemma exhibits an alternative way of looking at

$$
\left[\begin{array}{cc}
A & V \\
-V^{*} & 0
\end{array}\right]
$$

as a $3 \times 3$ operator matrix acting on $\mathscr{H} \oplus \mathscr{H} \oplus \mathscr{H}$. There are some properties of operators in class $(S)$ that become more transparent by using this representation.

Lemma 3.3. There exists a unitary transformation

$$
L_{0}: \mathscr{H} \oplus \mathscr{H} \rightarrow \mathscr{H}
$$

such that $L_{0}(\mathscr{C} \oplus\{0\})=$ null $V^{*}, L_{0}(\{0\} \oplus \mathscr{C})=\operatorname{ran} V$, and for $L=$ $L_{0} \oplus 1: \mathscr{H} \oplus \mathscr{K} \oplus \mathscr{H} \rightarrow \mathscr{H} \oplus \mathscr{H}$ we have

$$
L *\left[\begin{array}{cc}
A & V \\
-V^{*} & 0
\end{array}\right] L=\left[\begin{array}{lll}
A_{11} & A_{12} & 0 \\
A_{21} & A_{22} & 1 \\
0 & -1 & 0
\end{array}\right],
$$

where

$$
\left[\begin{array}{ll}
A_{11} & A_{12} \\
A_{21} & A_{22}
\end{array}\right]=L_{0}^{*} A L_{0}
$$

Proof. Let $W \in \mathscr{L}(\mathscr{H})$ be any isometry such that ran $W=$ null $V^{*}$, and define $L_{0}$ by $L_{0 \mid \mathscr{C} \oplus\{0\}}=W, L_{0 \mid\{0\} \oplus \mathscr{C}}=V$. It is clear that $L_{0}$ satisfies the required conditions.

Sometimes we can exhibit invariant subspaces of

$$
\left[\begin{array}{cc}
A & V \\
-V^{*} & 0
\end{array}\right]
$$

without being able to say anything about the invariant subspaces of the operator $A$.

THeOREM 4. Let $B \in \mathscr{L}(\mathscr{H})$ such that $B$ commutes with $V V^{*}$ and its essential spectrum $E(B) \subset D_{+}=\{\lambda \in C:|\lambda|<1, \operatorname{Im} \lambda>0\}$. Then

$$
\left[\begin{array}{cc}
B & V \\
-V^{*} & 0
\end{array}\right]
$$

is in $(E D)$, and hence there exists $\varepsilon>0$ such that for every $R \in \mathscr{L}(\mathscr{H})$, $\|R\|<\varepsilon$ and every compact $K \in \mathscr{L}(\mathscr{H})$, the operator 


$$
\left[\begin{array}{cc}
B+R+K & V \\
-V^{*} & 0
\end{array}\right]
$$

has two infinite dimensional, complementary hyperinvariant subspaces (a similar statement holds if $D_{+}$is replaced by the set

$$
\left.D_{-}=\{\lambda \in \boldsymbol{C} ;|\lambda|<1, \operatorname{Im} \lambda<0\}\right) \text {. }
$$

Proof. This is an immediate consequence of Lemma 3.3 and [10], Theorem 10.

In the last theorem the condition $B V V^{*}=V V^{*} B$ is actually placed there to facilitate the calculation of the spectrum of

$$
T=\left[\begin{array}{cc}
B & V \\
-V^{*} & 0
\end{array}\right]
$$

while $E(B) \subset D_{+}$is one of the simplest conditions to require in order that $\hat{E}(T)$ be disconnected. In fact, in that case $\hat{E}(T)$ has at least three components.

In the next section we impose upon ourselves the task of giving an example of an operator

$$
\left[\begin{array}{cc}
A & V \\
-V^{*} & 0
\end{array}\right]
$$

in $(E D)$, such that $A$ does not commute with $V V^{*}$ and the spectrum $\Sigma(A)$ of $A$ is contained in the real axis.

4. Examples of operators in $(E D) \cap(S)$. We base the results of the present paragraph upon an important algebraic lemma of Kaplansky ([ 9], Lemma 5). In order to state such a result we need to recall some definitions.

A weakly closed *-subalgebra of $\mathscr{L}(\mathscr{H})$ with identity will be called a Von Neumann algebra acting on $\mathscr{H}$. We denote by $\mathscr{C}_{2}(\mathscr{A})$ the ring of $2 \times 2$ matrices whose entries lie in the Von Neumann algebra $\mathscr{A}$. Any Von Neumann algebra which is unitarily equivalent to $\mathscr{C}_{2}(\mathscr{A})$, for some abelian Von Neummann algebra $\mathscr{A}$, will be called a 2-homogeneous Von Neumann algebra. A binormal ring is a Von Neumann algebra unitarily equivalent to the direct sum $\mathscr{C l}_{2}(\mathscr{A}) \oplus \mathscr{\mathscr { B }}$, for some abelian Von Neumann algebras $\mathscr{A}$ and $\mathscr{B}$ ([3], Theorem 1 and 2).

Lemma (Kaplansky). The Von Neumann algebra generated by any two projections in $\mathscr{L}(\mathscr{H})$ is a binormal ring. 
COROLlaRY 4.1. If $P$ is any projection in $\mathscr{L}(\mathscr{H})$, then the operator

$$
\left[\begin{array}{cc}
P & V \\
-V^{*} & 0
\end{array}\right]
$$

has a nontrivial reducing subspace.

Proof. Since the Von Neumann algebra $\mathscr{R}$ generated by $P$ and $V V^{*}$ is a binormal ring and $\mathscr{H}$ is infinite dimensional, the commutant of $\mathscr{R}$ is not trivial. Hence, there exists a nontrivial projection $R \in \mathscr{L}(\mathscr{H})$ commuting with $P$ and $V V^{*}$. Now, the corollary follows from Lemma 3.2 .

In what follows we summarize some known facts about binormal rings ([3]) that will be needed in the proof of the next theorem.

Given a binormal ring $\mathscr{R}$ there exists a projection $F$ in the center $\mathscr{Z}$ of $\mathscr{R}$ which is maximal among all those projections $R \in \mathscr{Z}$ such that $R \mathscr{R} R$ is an abelian Von Neumann algebra. Likewise, the projection $G=1-F$ in $\mathscr{F}$ is maximal among all those projections $R \in \mathscr{Z}$ such that $R \mathscr{R} R$ is a 2-homogeneous Von Neumann algebra. $F$ and $G$ are called maximal abelian and maximal 2-homogeneous central projections of $\mathscr{R}$, respectively. It follows that $\mathscr{B}$ acts on ran $F$ and $\mathscr{C}_{2}(\mathscr{A})$ acts on ran $G$.

Let $\mathscr{A}$ be an abelian Von Neumann algebra. There exists an extremely disconnected, compact, Hausdorff (Stonian) space $X$ such that $\mathscr{A}$ is *-isometrically isomorphic to the Banach algebra $C(X)$ of all continuous complex valued functions on $X$. Furthermore, it can be proved ([3], Theorem 4) that the algebra $\mathscr{L}_{2}(\mathscr{A})$ is *-isometrically isomorphic to the Banach algebra $\mathscr{M}_{2}(X)$ of all continuous functions from $X$ to $\mathscr{C}_{2}(C)$ (the ring of $2 \times 2$ complex matrices) with the supremum norm and algebraic pointwise operations. Under this isomorphism, every element $S \in \mathscr{l}_{2}(\mathscr{A})$ can be represented as a function $\widehat{S} \in \mathscr{C l}_{2}(X)$. As an immediate consequence of the above *-isometric isomorphism between $\mathscr{C}_{2}(\mathscr{A})$ and $\mathscr{C}_{2}(X)$ we conclude that the following equivalences are valid:

$S \in \mathbb{C l}_{2}(\mathscr{A})$ is invertible if and only if $\hat{S} \in \mathscr{C l}_{2}(X)$ is invertible, if and only if $\widehat{S}(x)$ is invertible in $\mathscr{C}_{2}(C)$, for every $x \in X$.

Therefore $S$ is invertible in $\mathscr{C}_{2}(\mathscr{A})$ if and only if the determinant $\operatorname{det} \widehat{S}(x) \neq 0$, for every $x \in X$. On the other hand, it can also be proved ([5], Theorem 2) that given any $\mu, \nu \in C(X)$ such that, for each $x \in X$, $\mu(x)$ and $\nu(x)$ are the two possible eigenvalues of $\hat{S}(x)$, then there exists a unitary $U \in \mathscr{C}_{2}(\mathscr{C})$ and $\rho \in C(X)$ such that

$$
\hat{U}(x) \hat{S}(x) \hat{U}^{*}(x)=\left[\begin{array}{cc}
\mu(x) & \rho(x) \\
0 & \nu(x)
\end{array}\right]
$$


for every $x \in X$, and hence $U S U^{*}$ is in the upper triangular form.

Theorem 5. Let $P$ be any projection in $\mathscr{L}(\mathscr{H})$. Then the operator

$$
T=\left[\begin{array}{cc}
P & V \\
-V^{*} & 0
\end{array}\right]
$$

is in $(E D)$. Furthermore, $\Sigma(T) \subset p^{-1}([0,1])$, where $p$ is the cubic polynomial $p(\lambda)=-\lambda^{3}+\lambda^{2}-\lambda+1$.

Proof. It can be easily checked that

$$
p(T)=\left[\begin{array}{cr}
1-\left(P-V V^{*}\right)^{2} & 0 \\
0 & V^{*} P V
\end{array}\right] .
$$

Since $\left(P-V V^{*}\right)^{2}$ and $V^{*} P V$ are positive contractions, so is $p(T)$ and hence from the spectral mapping theorem $\Sigma(T) \subset p^{-1}([0,1])$. It is easy to verify that the set $p^{-1}([0,1])$ consists of three $\operatorname{arcs} C_{j}, j=-1,0,1$, where $C_{0}=[0,1], C_{1}$ is an arc in the open upper half plane whose extremes are the points $i$ and $(1+i \sqrt{3 / 2})$, and $C_{-1}$ is the conjugate set of $C_{1}$. To complete the proof of the theorem it suffices to show that $E(T) \cap C_{ \pm 1} \neq \varnothing$.

From [7], Problem 56, we see that for $\lambda \neq 0, T-\lambda$ is not invertible if and only if the formal determinant $\Delta(\lambda)$ of $T-\lambda$, that is

$$
\Delta(\lambda)=\lambda^{2}-\lambda P+V V^{*}
$$

is not invertible. Set $Q=V V^{*}$. Then $\lambda \in \Sigma(T) \cap\left[C_{1} \cup C_{-1}\right]$ if and only if

$$
\lambda^{2}-\lambda P+Q
$$

is not invertible.

Let $\mathscr{R}=\mathscr{L}_{2}(\mathscr{A}) \oplus \mathscr{B}$ (where $\mathscr{A}$ and $\mathscr{B}$ are abelian) be the binormal ring generated by $P$ and $Q$, and assume that $\mathscr{G}$ is the Hilbert space on which $\mathscr{R}$ acts. Since $Q$ is infinite dimensional, $\mathscr{C}$ is also infinite dimensional. Now let $F$ and $G$ be the maximal abelian and maximal 2-homogeneous central projections of $\mathscr{R}$ respectively, and let $P_{F}\left(Q_{F}\right)$ be the restriction of $P$ (of $Q$ ) to $F \mathscr{C}$ and let

$$
\Delta_{F}(\lambda)=\lambda^{2}-\lambda P_{F}+Q_{F} .
$$

If $G$ is finite dimensional, then $F$ and $Q_{F}$ are infinite dimensional. In this case it is easy to prove either $\Delta_{F}( \pm i)$, or $\Delta_{F}(1 / 2(1 \pm \sqrt{3 i})$ have an infinite dimensional null space. Therefore $\Delta(\lambda)$ has the same property, for such values of $\lambda$ and hence ([10], Lemma 4.1) $E(T) \cap C_{j} \neq \varnothing$, 
$j= \pm 1$. Therefore we can restrict our discussion to the case $G$ infinite dimensional. Furthermore, with a small abuse of notation, we can suppose $G=1$ and that $P$ and $Q$ generate the 2-homogeneous Von Neumann algebra $\mathscr{A}_{2}(\mathscr{A})$. In this situation the set

$$
\{x \in X: \hat{P}(x) \hat{Q}(x) \neq \hat{Q}(x) \hat{P}(x)\}
$$

is dense in $X$, and hence the trace of $\hat{P}(x)$ and $\hat{Q}(x)$ are identically one on $X$. Since for each $x \in X$ the eigenvalues of the matrix $\hat{Q}(x)$ are 0 and 1 , we can apply a unitary equivalence in $\mathscr{L}_{2}(\mathscr{A})$ so that the projection $Q$ takes the form

$$
\left[\begin{array}{ll}
1 & 0 \\
0 & 0
\end{array}\right]
$$

We assume that this has already been done and we keep the same notation for $P$ and $Q$. Setting

$$
\hat{P}(x)=\left[\begin{array}{ll}
\alpha(x) & \gamma(x) \\
\gamma(x) & \beta(x)
\end{array}\right]
$$

we see that $\alpha(x)+\beta(x)=1, \alpha(x) \beta(x)-|\gamma(x)|^{2}=0$ and $0 \leqq \alpha(x) \leqq 1$ for every $x \in X$. We use the last condition, equation $\left(^{*}\right)$ and the fact that $\Delta(\lambda)$ is invertible if and only if $[\Delta(\lambda)]^{\wedge}(x)$ is invertible, for every $x \in X$, to conclude that $\lambda \in \Sigma(T) \cap\left[C_{1} \cup C_{-1}\right]$ if and only if, for some $x \in X$

$$
-\lambda^{3}+\lambda^{2}-\lambda+1-\alpha(x)=p(\lambda)-\alpha(x)=0 .
$$

If $\alpha$ takes infinitely many values on $X$, then from $\left.{ }^{* *}\right) C_{ \pm 1}$ contains infinitely many points, and hence (using again [10], Lemma 4.1) $E(T) \cap C_{ \pm 1} \neq \varnothing$. Therefore we can suppose that there are open compact subsets $X_{0}, \cdots, X_{n}$ of $X$ such that $\alpha$ is a constant $\alpha(x)=\alpha_{j}$, for $x \in X_{j}, 0 \leqq j \leqq n$. Let $G_{j}$ be the central projection corresponding to the sets $X_{j}$, that is

$$
\widehat{G}_{j}=\chi_{x_{j}}\left[\begin{array}{ll}
1 & 0 \\
0 & 1
\end{array}\right]
$$

where $\chi_{x_{j}}$ is the characteristic function of $X_{j}$. Since $\mathscr{G}$ is infinite dimensional, one of $G_{j}$, say $G_{0}$ is an infinite dimensional range projection. Let $P_{0}=P G_{0}$ and $Q_{0}=Q G_{0}$ (note that in the algebra $\left[G_{0} \mathscr{C}_{2}(\mathscr{A})\right]_{\mid G_{0},}$ we have $\left.Q_{0}=\left[\begin{array}{ll}1 & 0 \\ 0 & 0\end{array}\right]\right)$. The final step in this proof consists of showing that the null space of $\lambda_{j}^{2}-\lambda_{j} P_{0}+Q_{0}$ is infinite dimensional, where $\lambda_{j} \in C_{j}, j= \pm 1$ are roots of $p(\lambda)=\alpha_{0}$. From 
this it will follow that $\lambda_{1}$ and $\lambda_{-1}$ are eigenvalues of infinite multiplicity of $T$, and hence that $E(T) \cap C_{ \pm 1} \neq \varnothing$.

Let $\beta_{0}=1-\alpha_{0}=\beta(x), \delta_{0}=\sqrt{\alpha_{0} \beta_{0}}=|\gamma(x)|$, for every $x \in X_{0}$. If $\delta_{0} \neq 0$ we define the unitary $W \in \mathscr{L}_{2}(\mathscr{A})$ by

$$
\begin{aligned}
& \hat{W}(x)=\left[\begin{array}{ll}
\frac{\gamma(x)}{|\gamma(x)|} & 0 \\
0 & 0
\end{array}\right], \quad x \in X_{0}, \\
& \hat{W}(x)=\left[\begin{array}{ll}
1 & 0 \\
0 & 1
\end{array}\right], \quad x \in X-X_{0},
\end{aligned}
$$

then

$$
\hat{W}(x) \hat{P}_{0}(x) \hat{W}^{*}(x)=\left[\begin{array}{cc}
\alpha_{0} & \delta_{0} \\
\delta_{0} & \beta_{0}
\end{array}\right], \quad x \in X_{0}
$$

In any case we can assume that $\hat{P}_{0}(x)$ is a constant matrix, for every $x \in X_{0}$. Since $G_{0} \mathscr{G}$ is infinite dimensional, it follows that, for $j= \pm 1$

$$
\lambda_{j}^{2}-\lambda_{j} P_{0}+Q_{0}
$$

is a singular two by two scalar operator matrix acting in the direct sum of two copies of an infinite dimensional Hilbert space. Therefore

$$
\lambda_{j}^{2}-\lambda_{j} P_{0}+Q_{0}
$$

has an infinite dimensional null space, $j= \pm 1$.

REMARK. The above theorem can be extended without difficulties to operators of the form

$$
\left[\begin{array}{cc}
\theta P & V \\
-V^{*} & 0
\end{array}\right]
$$

where $|\theta|=1$ and $P$ is any projection in $\mathscr{L}(\mathscr{C})$. In this case the polynomial $p(\lambda)$ has to be replaced by $p_{0}(\lambda)=(1 / \theta)\left(-\lambda^{3}+\theta \lambda^{2}-\lambda+\theta\right)$. For $\theta=i$ a much simpler proof can be given ([10], Theorem 9). In another direction, Theorem 5 can be extended, using similar arguments, to positive contractions $A$ satisfying: $A$ and $V V^{*}$ generate a binormal ring. However this is not a significant generalization since few positive contractions, other than projections, generate together with $V V^{*}$ a binormal ring. Nevertheless, we believe that such a condition should not be essential in proving that

$$
\left[\begin{array}{cc}
A & V \\
-V^{*} & 0
\end{array}\right]
$$


is in $(E D)$, for a positive contraction $A$. Observe that the spectrum of

$$
\left[\begin{array}{cc}
1+V V^{*} & V \\
-V^{*} & 0
\end{array}\right]
$$

is the singleton $\{1\}$. Therefore we cannot expect that

$$
\left[\begin{array}{cc}
A & V \\
-V^{*} & 0
\end{array}\right]
$$

be in $(E D)$, for any positive operator $A$.

\section{REFERENCES}

1. N. Aronszajn, and K. T. Smith, Invariant subspaces of completely continuous operators, Ann. Math., 60 (1954), 345-350.

2. A. R. Bernstein and A. Robinson, Solution of the invariant subspace problem of K. T. Smith and P. R. Halmos, Pacific J. Math., 16 (1966), 421-431.

3. A. Brown, The unitary equivalence of binormal operators, Amer. J. Math., 76, No. 2 (1954), 414-434.

4. A. Brown and C. Pearcy, Structure of Commutators of operators, Ann. Math., 82 (1965), 112-127.

5. D. Deckard and C. Pearcy, On matrices over the ring of continuous complex valued functions on a Stonian space, Proc. Amer. Math. Soc., 14, No. 2, (1963), 322-328.

6. R. G. Douglas and C. Pearcy, On a topology for invariant subspaces, J. Functional Analysis, 2, No. 3 (1968), 323-341.

7. P. Halmos, A Hilbert Space Problem Book, Van Nostrand (1967), Princeton.

8. - Shifts on Hilbert spaces, J. reine angew, Math., 208, pp. 102-112.

9. I. Kaplansky, Symmetry on Banach algebras, Proc. Amer. Math. Soc., 3 (1952), 396-399.

10. N. Salinas, Operators with essentially disconnected spectrum, to appear in Acta Sci. Math. (Szeged).

Received January 19, 1971. This paper is a part of the author's doctoral dissertation written at the University Michigan. The author wishes to express his sincere gratitude to Prof. Carl Pearcy for his helpful suggestions during the preparation of this paper.

The University of MichigaN 



\section{PACIFIC JOURNAL OF MATHEMATICS}

\section{EDITORS}

\author{
H. SAMELSON \\ Stanford University \\ Stanford, California 94305 \\ C. R. HOBBY \\ University of Washington \\ Seattle, Washington 98105
}

\section{J. DuGundJI}

Department of Mathematics University of Southern California Los Angeles, California 90007

RICHARD ARENS

University of California

Los Angeles, California 90024

\section{ASSOCIATE EDITORS}
E. F. BECKENBACH
B. H. NeumanN
F. WOLF
K. YOSHIDA

\section{SUPPORTING INSTITUTIONS}

\author{
UNIVERSITY OF BRITISH COLUMBIA \\ CALIFORNIA INSTITUTE OF TECHNOLOGY \\ UNIVERSITY OF CALIFORNIA \\ MONTANA STATE UNIVERSITY \\ UNIVERSITY OF NEVADA \\ NEW MEXICO STATE UNIVERSITY \\ OREGON STATE UNIVERSITY \\ UNIVERSITY OF OREGON \\ OSAKA UNIVERSITY
}

\author{
UNIVERSITY OF SOUTHERN CALIFORNIA \\ STANFORD UNIVERSITY \\ UNIVERSITY OF TOKYO \\ UNIVERSITY OF UTAH \\ WASHINGTON STATE UNIVERSITY \\ UNIVERSITY OF WASHINGTON \\ AMERICAN MATHEMATICAL SOCIETY \\ NAVAL WEAPONS CENTER
}

The Supporting Institutions listed above contribute to the cost of publication of this Journal, but they are not owners or publishers and have no responsibility for its content or policies.

Mathematical papers intended for publication in the Pacific Journal of Mathematics should be in typed form or offset-reproduced, (not dittoed), double spaced with large margins. Underline Greek letters in red, German in green, and script in blue. The first paragraph or two must be capable of being used separately as a synopsis of the entire paper. The editorial "we" must not be used in the synopsis, and items of the bibliography should not be cited there unless absolutely necessary, in which case they must be identified by author and Journal, rather than by item number. Manuscripts, in duplicate if possible, may be sent to any one of the four editors. Please classify according to the scheme of Math. Rev. Index to Vol. 39. All other communications to the editors should be addressed to the managing editor, Richard Arens, University of California, Los Angeles, California, 90024.

50 reprints are provided free for each article; additional copies may be obtained at cost in multiples of 50 .

The Pacific Journal of Mathematics is published monthly. Effective with Volume 16 the price per volume (3 numbers) is $\$ 8.00$; single issues, $\$ 3.00$. Special price for current issues to individual faculty members of supporting institutions and to individual members of the American Mathematical Society: $\$ 4.00$ per volume; single issues $\$ 1.50$. Back numbers are available.

Subscriptions, orders for back numbers, and changes of address should be sent to Pacific Journal of Mathematics, 103 Highland Boulevard, Berkeley, California, 94708.

PUBLISHED BY PACIFIC JOURNAL OF MATHEMATICS, A NON-PROFIT CORPORATION

Printed at Kokusai Bunken Insatsusha (International Academic Printing Co., Ltd.), 270, 3-chome Totsuka-cho, Shinjuku-ku, Tokyo 160, Japan. 


\section{Pacific Journal of Mathematics}

\section{Vol. 42, No. $2 \quad$ February, 1972}

Stephen Richard Bernfeld, The extendability of solutions of perturbed scalar differential equations ................................. 277

James Edwin Brink, Inequalities involving $f_{-} p$ and $f^{(n)}{ }_{q}$ for $f$ with $n$

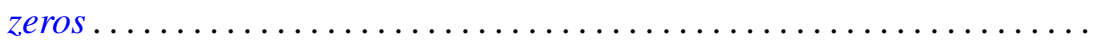

Orrin Frink and Robert S. Smith, On the distributivity of the lattice of filters of a groupoid

Donald Goldsmith, On the density of certain cohesive basic sequences .... 323

Charles Lemuel Hagopian, Planar images of decomposable continua . . . . . 329

W. N. Hudson, A decomposition theorem for biadditive processes ........ 333

W. N. Hudson, Continuity of sample functions of biadditive processes......

Masako Izumi and Shin-ichi Izumi, Integrability of trigonometric series.



H. M. Ko, Fixed point theorems for point-to-set mappings and the set of

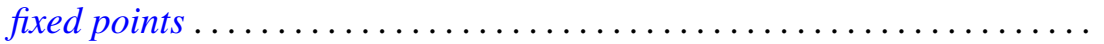

Gregers Louis Krabbe, An algebra of generalized functions on an open interval: two-sided operational calculus ...

Thomas Latimer Kriete, III, Complete non-selfadjointness of almost selfadjoint operators.................................

Shiva Narain Lal and Siya Ram, On the absolute Hausdorff summability of a Fourier series .

Ronald Leslie Lipsman, Representation theory of almost connected groups...

James R. McLaughlin, Integrated orthonormal series.... . .

H. Minc, On permanents of circulants.

Akihiro Okuyama, On a generalization of $\Sigma$-spaces.....

Norberto Salinas, Invariant subspaces and operators of class $(S)$

James D. Stafney, The spectrum of certain lower triangular matrices as operators on the $l_{p}$ spaces .......................

Arne Stray, Interpolation by analytic functions

$\mathrm{Li} \mathrm{Pi} \mathrm{Su}$, Rings of analytic functions on any subset of the complex plane.

R. J. Tondra, A property of manifolds compactly equivalent to compact manifolds.... 